\title{
Medical insurance and healthcare utilization among the middle-aged and elderly in China: evidence from the China health and retirement longitudinal study 2011, 2013 and 2015
}

Yue Zhou' ${ }^{1}$, Haishaerjiang Wushouer ${ }^{2,3}$, Daniel Vuillermin ${ }^{4}$, Bingyu $\mathrm{Ni}^{1}$, Xiaodong Guan ${ }^{1,5,6}$ and Luwen Shi ${ }^{1,5^{*}}$ (D)

\begin{abstract}
Background: In response to China's rapidly aging population and increasing healthcare service demands, the Chinese government is developing a universal medical insurance system. This study aimed to assess healthcare utilization patterns and analyze the impacts of medical insurance schemes on healthcare utilization among the middle-aged and elderly in China.

Methods: Data was extracted from the China Health and Retirement Longitudinal Study in 2011, 2013 and 2015. Healthcare utilization was measured by outpatient and inpatient service utilization. Univariate analysis was deployed to examine the impacts of different medical insurance schemes on healthcare utilization. The factors associated with healthcare utilization were estimated using a random-effects logistic regression model.

Results: During the study period, the number of individuals involved was 17,250, 18,195 and 19,842, respectively. The proportion of individuals who received outpatient service was 18.6, 20.7 and $18.7 \%$ and those who used inpatient service was 9.6, 13.8 and 14.3\%, respectively. We identified that medical insurance was a major protective factor for improving healthcare utilization but different medical insurance schemes exerted various impacts on the middle-aged and the elderly.
\end{abstract}

Conclusions: Despite the growing population coverage, the Chinese government should make every effort to bridge the gap among people with different medical insurance schemes. Further evaluation is needed to assess whether the expanded medical insurance schemes could protect the middle-aged and elderly households from catastrophic health expenditure.

Keywords: Healthcare utilization, Medical insurance, Aging population

\footnotetext{
* Correspondence: shilu@bjmu.edu.cn

'Department of Pharmacy Administration and Clinical Pharmacy, School of

Pharmaceutical Sciences, Peking University, No.38 Xueyuan Road, Haidian

District, Beijing 100191, China

${ }^{5}$ International Research Center for Medicinal Administration, Peking

University, No.38 Xueyuan Road, Haidian District, Beijing 100191, China

Full list of author information is available at the end of the article
}

(c) The Author(s). 2020 Open Access This article is licensed under a Creative Commons Attribution 4.0 International License, which permits use, sharing, adaptation, distribution and reproduction in any medium or format, as long as you give appropriate credit to the original author(s) and the source, provide a link to the Creative Commons licence, and indicate if changes were made. The images or other third party material in this article are included in the article's Creative Commons licence, unless indicated otherwise in a credit line to the material. If material is not included in the article's Creative Commons licence and your intended use is not permitted by statutory regulation or exceeds the permitted use, you will need to obtain permission directly from the copyright holder. To view a copy of this licence, visit http://creativecommons.org/licenses/by/4.0/ The Creative Commons Public Domain Dedication waiver (http://creativecommons.org/publicdomain/zero/1.0/) applies to the data made available in this article, unless otherwise stated in a credit line to the data. 


\section{Background}

Aging populations is becoming a prominent issue facing many countries around the world [1]. In 2017, there were approximately 54 million people aged 45 and above in mainland China, which accounted for up to $40.2 \%$ of the population [2]. People have a longer lifespan but also higher morbidity rates due to non-communicable diseases (NCD), such as hypertension, diabetes and cardiovascular disease [3, 4]. In China, an aging population has brought more than 300 million NCD patients across the country which led to increasing healthcare utilization and further straining healthcare services [4-6]. Therefore, providing high-quality healthcare services is of great need [1, 7].

While China's Opening up and Reform policies have lifted millions of people out of poverty, there is also a growing gulf between the rich and the poor $[8,9]$. Low-income groups suffer greater challenges in accessing healthcare services and are more likely to be impoverished due to the health expenditures. This exacerbates the disparities between the rich and the poor in healthcare utilization [10]. In response to these problems, the Chinese government develops a universal medical insurance system, including the Urban Employees Basic Medical Insurance (UEBMI), the New Rural Cooperative Medical Insurance Scheme (NRCMS) and the Urban Residents' Basic Medical Insurance (URBMI). Specifically, UEBMI, initiated for urban employees and retired employees in 1998 , is a mandatory medical insurance scheme. NRCMS, launched in 2003, is a voluntary medical insurance scheme targeting rural populations. In 2007, URBMI was formulated for urban residents without formal employment who could enroll voluntarily. UEBMI is mainly financed by the payroll taxes from beneficiaries. NRCMS and URBMI are financed by the government and premiums, with the government subsidies accounted for majority of the financing [11]. Then, along with the crucial health system reforms in 2009, great strides have been made to consolidate NRCMS and URBMI into the Urban and Rural Resident Medical Insurance. From 2011 to 2015, the premium for NRCMS and URBMI significantly increased and the benefit packages expanded. In general, the list of medical services eligible for reimbursement for NRCMS and URBMI is shorten than that of UEBMI and the reimbursement rate is even lower [11]. The medical insurance schemes mentioned together with private medical insurances (PMI) comprise the national medical insurance system in China [11]. Consequently, near-universal medical insurance coverage has been achieved, from only $85 \%$ ( 1.13 billion) of the Chinese population covered in 2008 to $95 \%$ (1.28 billion) in 2011 and 97\% (1.33 billion) in 2015 [12-14].
Previous studies found that insurances would lower the barriers for individuals to visit a doctor or have physical examinations. As UEBMI individuals provided the most generous benefit packages, individuals covered by URBMI were more likely to use healthcare services, especially inpatient service [15-18]. However, great strides were made in improving the health system in China and the benefit packages of NRCMS and URBMI were improved. We hypothesized that the promoted medical insurance system could narrow the gaps in healthcare utilization among individuals covered by different medical insurance schemes. Hence, we conducted this study based on a three-year panel data which could track variations in healthcare utilization over time to assess healthcare utilization patterns and analyze the impacts of the medical insurance schemes on healthcare utilization among the middle-aged and elderly in China.

\section{Methods \\ Data source}

Data were extracted from the China Health and Retirement Longitudinal Study (CHARLS) (http://charls.pku. edu.cn/) in 2011, 2013 and 2015 [19]. The CHARLS national surveys targeted population aged 45 and above in 150 counties from 28 provinces in China. In our study, the panel data of the 2011 CHARLS Wave 1 (Baseline), the 2013 CHARLS Wave 2 and the 2015 CHARLS Wave 3 were used to describe the patterns of healthcare utilization and evaluate the impacts of medical insurance schemes on healthcare utilization among the middleaged and elderly in China.

\section{Study variables}

The dependent variable was healthcare utilization measured by outpatient and inpatient service utilization. Outpatient service utilization was defined as visiting but without admission to medical facilities, including general hospitals, specialized hospitals, traditional Chinese medicine hospitals, community healthcare centers, township hospitals, health care posts or village clinic/private clinics in the last 4 weeks for treatment. Inpatient service utilization was defined as being admitted within the last year to a general hospital, specialized hospital, Chinese medicine hospital, community healthcare center, township hospital or health care post [19].

Based on previous literatures, the independent variables included economic status (total household annual expenditure per capita), medical insurance schemes, individual characteristics (gender, age, marital status, educational status, registration place, region), health status and functioning (self-reported health, chronic diseases, depression) and family information (household size) $[17,20,21]$. (Specific information in Table 1). 
Table 1 Description of variables in 2011, 2013 and $2015^{\text {a }}$

\begin{tabular}{|c|c|c|c|}
\hline \multirow[t]{2}{*}{ Variables } & $\begin{array}{l}2011 \\
N=17,250\end{array}$ & $\begin{array}{l}2013 \\
N=18,195\end{array}$ & $\begin{array}{l}2015 \\
N=19,842\end{array}$ \\
\hline & $n(\%)^{b}$ & n (\%) & n (\%) \\
\hline \multicolumn{4}{|c|}{ Medical Insurance Schemes } \\
\hline NRCMS & $12,285(72.2)$ & $12,879(72.1)$ & $13,208(68.8)$ \\
\hline UEBMI & $2101(12.4)$ & $2420(13.6)$ & $2810(14.6)$ \\
\hline URBMI & $948(5.6)$ & $1284(7.2)$ & $1361(7.1)$ \\
\hline PMI & $540(3.2)$ & $555(3.1)$ & $185(1.0)$ \\
\hline NONE & $1137(6.7)$ & $726(4.1)$ & $1634(8.5)$ \\
\hline
\end{tabular}

Economic Status (Average Total Household Annual Expenditure Per Capita in 2011, 2013 and 2015/US Dollars) ${ }^{c}$

Quintile $1(255.1,371.0,409.7)$
Quintile $2(520.3,749.7,869.5)$
Quintile $3(803.1,1129.3,1368.8)$
Quintile $4(1260.0,1740.9,2145.3)$
Quintile $5(3318.9,4396.0,6052.2)$

\section{Gender}

\author{
Female
}

Male

Age

$$
\begin{aligned}
& {[45,55]} \\
& (55,65]
\end{aligned}
$$

$(65,+\infty)$

\section{Marital Status}

Married or Cohabitated

Divorced, Widowed, Never Married, Separated

\section{Education Status}

Below Primary School

Primary School

Secondary School

High School and Above

\section{Registration Place}

Non-rural areas

Rural Areas

\section{Region}

\section{Eastern \\ Central}

Western

\section{Chronic Diseases $^{d}$}

No

Yes

\section{Self-reported Health}

Good
Fair
Poor

$$
\begin{aligned}
& 2891(20.1) \\
& 2888(20.1) \\
& 2882(20.1) \\
& 2847(19.8) \\
& 2866(19.9)
\end{aligned}
$$

8407 (48.7)

$8841(51.3)$

$6895(40.0)$

6117 (35.5)

$4238(24.6)$

$14,990(87.0)$

$2248(13.0)$

4765 (27.7)

$6715(39.0)$

3555 (20.6)

$2198(12.8)$

6985 (40.5)

$10,265(59.5)$

5990 (34.7)

5654 (32.8)

$5603(32.5)$

5566 (32.5)

$11,573(67.5)$

3409 (27.7)

$5790(47.0)$

$3119(25.3)$
$2400(20.2)$

2385 (20.1)

$2377(20.0)$

2366 (20.0)

2360 (19.9)

8816 (48.5)

9378 (51.5)

6705 (36.9)

$6553(36.0)$

4937 (27.1)

$15,792(86.8)$

2396 (13.2)

4825 (26.5)

7161 39.4)

3822 (21.0)

2375 (13.1)

$7348(40.4)$

10,843 (59.6)

$6290(34.6)$

$5954(32.7)$

$5940(32.7)$

6384 (35.4)

11,657 (64.6)

4407 (25.6)

$9032(52.5)$

3765 (21.9)
$12,196(61.9)$

4705 (25.4)

2747 (20.6)

2701 (20.3)

2644 (19.9)

2620 (19.7)

2606 (19.6)

9653 (48.7)

$10,181(51.3)$

7410 (37.4)

6742 (34.0)

5690 (28.7)

17,140 (86.4)

2696 (13.6)

5021 (25.4)

8673 (43.8)

3835 (19.4)

2274 (11.5)

8032 (40.5)

11,807 (59.5)

6957 (35.1)

6438 (32.5)

6442 (32.5)

9918 (53. 6)

3894 (21.0) 
Table 1 Description of variables in 2011, 2013 and $2015^{\text {a }}$ (Continued)

\begin{tabular}{|c|c|c|c|}
\hline \multirow[t]{2}{*}{ Variables } & $\begin{array}{l}2011 \\
N=17,250\end{array}$ & $\begin{array}{l}2013 \\
N=18,195\end{array}$ & $\begin{array}{l}2015 \\
N=19,842\end{array}$ \\
\hline & $n(\%)^{b}$ & n (\%) & n (\%) \\
\hline \multicolumn{4}{|l|}{ Depression } \\
\hline Mild & $12,062(70.4)$ & $13,661(75.7)$ & $14,467(73.4)$ \\
\hline Moderate & $4209(24.6)$ & $3761(20.9)$ & $4267(21.6)$ \\
\hline Severe & $868(5.1)$ & $619(3.4)$ & $985(5.0)$ \\
\hline \multicolumn{4}{|l|}{ Household Size } \\
\hline $1-2$ & $6274(36.4)$ & $6424(35.3)$ & $7972(40.2)$ \\
\hline $3-4$ & $5874(34.1)$ & $6349(34.9)$ & $9378(47.3)$ \\
\hline$\geq 5$ & $5102(29.6)$ & $5418(29.8)$ & 2489 (12.6) \\
\hline Outpatient Service Utilization & $3117(18.6)$ & $3653(20.7)$ & $3615(18.7)$ \\
\hline Inpatient Service Utilization & $1631(9.6)$ & $2486(13.8)$ & $2813(14.3)$ \\
\hline
\end{tabular}

NRCMS New Rural Cooperative Medical Insurance Scheme, UEBMI Urban Employees Basic Medical Insurance, URBMI Urban Residents' Basic Medical Insurance, PMI Private Medical Insurance

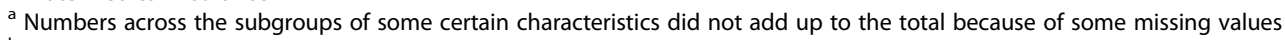

$\mathrm{b}$ " $\mathrm{n}$ " means the number of individuals in each group and "\%" means the proportion of individuals in each group

c Average total household annual expenditure per capita in 2011, 2013 and 2015 was 1228.2 US dollars, 1670.3 US dollars and 2138.9 US dollars, respectively

${ }^{d}$ Chronic diseases included hypertension, dyslipidemia, diabetes or high blood sugar, cancer or malignant tumor (excluding minor skin cancers), chronic lung diseases, such as chronic bronchitis, emphysema (excluding tumors, or cancer), liver disease (except fatty liver, tumors, and cancer), heart attack, stroke, kidney disease (except for tumor or cancer), stomach or other digestive disease (except for tumor or cancer), emotional, nervous, or psychiatric problems, memoryrelated disease, arthritis or rheumatism and asthma. In the surveys, people were asked whether they had ever been diagnosed with one of the listed chronic diseases and their responses were recorded

\section{Statistical analysis}

According to CHARLS website (http://charls.pku.edu. $\mathrm{cn} /$ index/en.html), new respondents will be included to guarantee the sample representativeness for some respondents may be lost or die. To solve this problem, we treated the data as unbalanced panel data.

\section{Descriptive and univariate analysis}

Descriptive analysis was used to summarize the characteristics of the individuals by expressing the results as the absolute frequencies and percentage. Consumer Price Index was used to adjust for inflation [22]. Total household annual expenditure per capita was converted into United States (US) dollars uniformly based on exchange rates in December 2011 (1 US dollar $=6.3281$ Yuan) to make the results more comparable to other published studies [23]. Then univariate analysis was used to examine the impacts of different factors on healthcare utilization by the Chi-squared test.

\section{Logistic regression analysis}

Random-effects logistic model for panel data was used to explore factors associated with healthcare utilization among the middle-aged and elderly in China. The model in our study could be defined by:

$$
\operatorname{Logit}\left(\operatorname{Pr}\left(y_{i t}=1\right)\right)=\alpha+\sum_{k=1}^{k} \beta_{k} x_{k i t}+\mu_{i t}
$$

$k=1,2,3 \ldots, \mathrm{N} ; t=2011,2013,2015$.
Where $y_{i t}$ is whether the individual $i_{t h}$ used healthcare services for the $t_{t h}$ time period, $\alpha$ refers to intercept, $\beta_{k}$ refers to coefficient vector which describe the effects of variables (If $\beta_{k}$ is over zero, the variable has a positive impact on $y_{i t}$ compared with the reference group and vice versa), $x_{k i t}$ denotes the value of the $k_{t h}$ variable on the $i_{t h}$ people for the $t_{t h}$ time period and $\mu_{i t}$ is the random error term.

Stata $^{\oplus}$ version 14.2 was used to perform the data analysis. $P<0.05$ was considered statistically significant.

\section{Results}

\section{Sample individual characteristics}

Table 1 showed the individual characteristics of the sample population. After excluding invalid data, the number of individuals involved in the three surveys was 17,250, 18,195 and 19,842, respectively. NRCMS covered most of the sampled individuals (72.2, 72.1 and 68.8\%), followed by UEBMI (12.4, 13.6 and 14.6\%). The proportion of individuals covered by no medical insurance scheme was $6.7,4.1$ and $8.5 \%$, respectively. The average total household annual expenditure per capita was 1254.4 US dollars, 1795.8 US dollars and 2378.4 US dollars, respectively. Female individuals accounted for 48.7, 48.5 and $48.7 \%$, respectively. The proportion of individuals over 65 years old was 24.6, 27.1 and $28.7 \%$. Individuals with chronic diseases accounted for 67.5, 64.6 and $61.9 \%$. The proportion of individuals with poor selfreported health also showed a decreasing trend, which was $25.3,21.9$ and $21.0 \%$. 
Table 2 Healthcare utilization patterns and univariate analysis in the middle-aged and elderly in 2011, 2013 and 2015

\begin{tabular}{|c|c|c|c|c|c|c|c|c|c|c|c|c|}
\hline \multirow[t]{3}{*}{ Variables } & \multicolumn{6}{|c|}{ Outpatient Service Utilization } & \multicolumn{6}{|c|}{ Inpatient Service Utilization } \\
\hline & \multicolumn{2}{|c|}{$\begin{array}{l}2011 \\
N=17,250\end{array}$} & \multicolumn{2}{|l|}{$\begin{array}{l}2013 \\
N=18,195\end{array}$} & \multicolumn{2}{|l|}{$\begin{array}{l}2015 \\
N=19,842\end{array}$} & \multicolumn{2}{|l|}{$\begin{array}{l}2011 \\
N=17,250\end{array}$} & \multicolumn{2}{|l|}{$\begin{array}{l}2013 \\
N=18,195\end{array}$} & \multicolumn{2}{|l|}{$\begin{array}{l}2015 \\
N=19,842\end{array}$} \\
\hline & $\mathrm{n}(\%)^{a}$ & $p$ & $\overline{n(\%)}$ & $p$ & $\mathrm{n}(\%)$ & $p$ & n (\%) & $p$ & n (\%) & $p$ & $\mathrm{n}(\%)$ & $p$ \\
\hline $\begin{array}{l}\text { Medical Insurance } \\
\text { Schemes }\end{array}$ & & 0.000 & & 0.000 & & 0.000 & & 0.000 & & 0.000 & & 0.000 \\
\hline NRCMS & $2371(19.6)$ & & $2706(21.4)$ & & $2528(19.4)$ & & $1129(9.2)$ & & 1753 (13.6) & & $1830(13.9)$ & \\
\hline UEBMI & $328(16.0)$ & & $476(20.2)$ & & $502(18.3)$ & & $277(13.2)$ & & $418(17.3)$ & & $485(17.3)$ & \\
\hline URBMI & $163(17.5)$ & & $236(18.8)$ & & $237(17.7)$ & & $101(10.7)$ & & $179(14.0)$ & & $220(16.2)$ & \\
\hline PMl & $100(18.7)$ & & $93(17.1)$ & & $36(19.8)$ & & $59(10.9)$ & & $59(10.7)$ & & $27(14.7)$ & \\
\hline NONE & $150(13.4)$ & & $114(16.0)$ & & $227(14.1)$ & & $59(5.2)$ & & $56(7.7)$ & & $195(12.0)$ & \\
\hline Economic Status & & 0.011 & & 0.001 & & 0.004 & & 0.000 & & 0.000 & & 0.000 \\
\hline Quintile 1 (Poorest) & $473(16.6)$ & & $433(18.2)$ & & $440(16.2)$ & & $176(6.1)$ & & $230(9.6)$ & & $278(10.1)$ & \\
\hline Quintile 2 & 565 (19.8) & & $482(20.5)$ & & 500 (18.8) & & $233(8.1)$ & & 279 (11.7) & & $283(10.5)$ & \\
\hline Quintile 3 & 528 (18.6) & & $468(20.1)$ & & 483 (18.6) & & 275 (9.6) & & 314 (13.2) & & 365 (13.8) & \\
\hline Quintile 4 & $552(19.7)$ & & $470(20.2)$ & & $471(18.3)$ & & $318(11.2)$ & & $349(14.8)$ & & $421(16.1)$ & \\
\hline Quintile 5 & $534(19.1)$ & & $532(23.2)$ & & $512(20.3)$ & & $378(13.2)$ & & 407 (17.3) & & $471(18.2)$ & \\
\hline Gender & & 0.000 & & 0.000 & & 0.000 & & 0.706 & & 0.736 & & 0.025 \\
\hline Female & 1325 (16.2) & & $1558(18.1)$ & & $1561(16.5)$ & & $803(9.6)$ & & 1214 (13.9) & & 1315 (13.7) & \\
\hline Male & $1792(20.8)$ & & $2095(23.1)$ & & $2054(20.7)$ & & $828(9.5)$ & & $1272(13.7)$ & & $1498(14.9)$ & \\
\hline Age & & 0.000 & & 0.000 & & 0.000 & & 0.000 & & 0.000 & & 0.000 \\
\hline$[45,55]$ & $1118(16.7)$ & & $1242(19.1)$ & & 1259 (17.4) & & $459(6.7)$ & & $661(10.0)$ & & $724(9.9)$ & \\
\hline$[55,65]$ & $1140(19.1)$ & & $1290(20.2)$ & & 1225 (18.6) & & $593(9.8)$ & & $900(13.8)$ & & $937(14.0)$ & \\
\hline$(65,+\infty)$ & 859 (20.9) & & $1121(23.4)$ & & 1131 (20.4) & & 579 (13.9) & & $925(19.1)$ & & $1152(20.5)$ & \\
\hline Marital Status & & 0.010 & & 0.000 & & 0.017 & & 0.108 & & 0.000 & & 0.000 \\
\hline Married or Cohabitated & 2667 (18.3) & & $3102(20.2)$ & & $3081(18.4)$ & & $1398(9.4)$ & & $2093(13.4)$ & & $2305(13.6)$ & \\
\hline $\begin{array}{l}\text { Divorced, Widowed, } \\
\text { Never Married, Separated }\end{array}$ & $450(20.6)$ & & $551(23.9)$ & & $534(20.4)$ & & $233(10.5)$ & & $393(16.7)$ & & $508(19.1)$ & \\
\hline Education Status & & 0.000 & & 0.000 & & 0.006 & & 0.043 & & 0.000 & & 0.000 \\
\hline Below Primary School & $979(21.0)$ & & $1057(22.5)$ & & $999(20.3)$ & & $482(10.2)$ & & $693(14.5)$ & & $824(16.5)$ & \\
\hline Primary School & $1252(19.1)$ & & $1475(21.1)$ & & $1564(18.4)$ & & $654(9.8)$ & & $1058(14.9)$ & & $1208(14.0)$ & \\
\hline Secondary School & $560(16.2)$ & & $713(19.3)$ & & $658(17.6)$ & & $314(8.9)$ & & $433(11.5)$ & & $502(13.2)$ & \\
\hline High School and Above & $325(15.4)$ & & $408(17.9)$ & & $390(17.9)$ & & $180(8.3)$ & & $301(12.9)$ & & $275(12.3)$ & \\
\hline Registration Place & & 0.000 & & 0.025 & & 0.003 & & 0.002 & & 0.000 & & 0.010 \\
\hline Non-rural areas & $1138(16.9)$ & & $1395(19.8)$ & & $1368(17.6)$ & & $715(10.4)$ & & $1081(15.0)$ & & $1194(15.1)$ & \\
\hline Rural areas & 1979 (19.7) & & $2258(21.2)$ & & $2247(19.3)$ & & $916(9.0)$ & & $1405(13.0)$ & & 1619 (13.8) & \\
\hline Region & & 0.085 & & 0.000 & & 0.000 & & 0.000 & & 0.000 & & 0.000 \\
\hline Eastern & $1032(17.7)$ & & $1154(18.8)$ & & $1178(17.4)$ & & $439(7.4)$ & & $693(11.2)$ & & $827(12.0)$ & \\
\hline Central & 1039 (18.8) & & $1142(19.8)$ & & $1137(18.0)$ & & $535(9.5)$ & & $806(13.7)$ & & $891(14.0)$ & \\
\hline Western & $1046(19.2)$ & & $1357(23.6)$ & & $1300(20.7)$ & & $657(11.9)$ & & $986(16.8)$ & & $1095(17.2)$ & \\
\hline Household Size & & 0.000 & & 0.000 & & 0.758 & & 0.000 & & 0.000 & & 0.000 \\
\hline $1-2$ & 1132 (18.6) & & $1244(20.0)$ & & $1452(18.7)$ & & $660(10.7)$ & & 981 (15.5) & & $1237(15.7)$ & \\
\hline $3-4$ & $977(17.1)$ & & $1244(20.2)$ & & 1695 (18.5) & & $484(8.3)$ & & $766(12.2)$ & & $1276(13.7)$ & \\
\hline$\geq 5$ & $1008(20.2)$ & & $1165(22.0)$ & & $468(19.2)$ & & $487(9.6)$ & & 739 (13.8) & & $300(12.1)$ & \\
\hline Chronic Diseases ${ }^{b}$ & & 0.000 & & 0.000 & & 0.000 & & 0.000 & & 0.000 & & 0.000 \\
\hline No & $545(10.0)$ & & $901(14.4)$ & & 1015 (13.7) & & $246(4.5)$ & & $532(8.4)$ & & 718 (9.6) & \\
\hline
\end{tabular}


Table 2 Healthcare utilization patterns and univariate analysis in the middle-aged and elderly in 2011, 2013 and 2015 (Continued)

\begin{tabular}{|c|c|c|c|c|c|c|c|c|c|c|c|c|}
\hline \multirow[t]{3}{*}{ Variables } & \multicolumn{6}{|c|}{ Outpatient Service Utilization } & \multicolumn{6}{|c|}{ Inpatient Service Utilization } \\
\hline & \multicolumn{2}{|l|}{$\begin{array}{l}2011 \\
N=17,250\end{array}$} & \multicolumn{2}{|l|}{$\begin{array}{l}2013 \\
N=18,195\end{array}$} & \multicolumn{2}{|l|}{$\begin{array}{l}2015 \\
N=19,842\end{array}$} & \multicolumn{2}{|l|}{$\begin{array}{l}2011 \\
N=17,250\end{array}$} & \multicolumn{2}{|l|}{$\begin{array}{l}2013 \\
N=18,195\end{array}$} & \multicolumn{2}{|l|}{$\begin{array}{l}2015 \\
N=19,842\end{array}$} \\
\hline & $\mathrm{n}(\%)^{a}$ & $p$ & $\mathrm{n}(\%)$ & $p$ & $\overline{n(\%)}$ & $p$ & $\mathrm{n}(\%)$ & p & $\overline{n(\%)}$ & p & $\mathrm{n}(\%)$ & p \\
\hline Yes & $2571(22.7)$ & & $2736(24.0)$ & & $2597(21.7)$ & & $1384(12.0)$ & & $1947(16.8)$ & & $2093(17.2)$ & \\
\hline Self-reported Health & & 0.000 & & 0.000 & & 0.000 & & 0.000 & & 0.000 & & 0.000 \\
\hline Good & $277(8.2)$ & & $483(11.1)$ & & $450(9.7)$ & & $141(4.2)$ & & $305(7.0)$ & & $308(6.6)$ & \\
\hline Fair & $952(16.8)$ & & $1741(19.7)$ & & $1742(17.8)$ & & $478(8.3)$ & & $1052(11.7)$ & & $1187(12.0)$ & \\
\hline Poor & 938 (30.9) & & $1264(34.6)$ & & $1212(32.1)$ & & 579 (18.6) & & $975(26.0)$ & & $1106(28.5)$ & \\
\hline Depression & & 0.000 & & 0.000 & & 0.000 & & 0.000 & & 0.000 & & 0.000 \\
\hline Mild & $1779(15.1)$ & & $2388(17.9)$ & & $2271(16.0)$ & & $978(8.2)$ & & $1620(12.0)$ & & $1742(12.1)$ & \\
\hline Moderate & $1074(25.9)$ & & $1028(27.9)$ & & $1014(24.3)$ & & $510(12.1)$ & & 703 (18.7) & & 808 (19.0) & \\
\hline Severe & $263(31.0)$ & & $221(37.0)$ & & 327 (33.9) & & $142(16.4)$ & & $156(25.3)$ & & $261(26.5)$ & \\
\hline
\end{tabular}

NRCMS New Rural Cooperative Medical Insurance Scheme, UEBMI Urban Employees Basic Medical Insurance, URBMI Urban Residents' Basic Medical Insurance, PMI Private Medical Insurance

a " $\mathrm{n}$ " means the number of individuals in each group who used healthcare service and "\%" means the proportion of individuals in each group who used healthcare service

${ }^{\mathrm{b}}$ Chronic diseases included hypertension, dyslipidemia, diabetes or high blood sugar, cancer or malignant tumor (excluding minor skin cancers), chronic lung diseases, such as chronic bronchitis, emphysema (excluding tumors, or cancer), liver disease (except fatty liver, tumors, and cancer), heart attack, stroke, kidney disease (except for tumor or cancer), stomach or other digestive disease (except for tumor or cancer), emotional, nervous, or psychiatric problems, memoryrelated disease, arthritis or rheumatism and asthma. In the surveys, people were asked whether they had ever been diagnosed with one of the listed chronic diseases and their responses were recorded

\section{Outpatient and inpatient service utilization}

As displayed in Table 1, in each of the three surveys of study, the proportion of individuals who received outpatient service was 18.6, 20.7 and $18.7 \%$ and who received inpatient service was 9.6, 13.8 and 14.3\%, respectively. Univariate analysis showed that significant differences existed between different medical insurance schemes $(p<0.05)$ as well as different economic status $(p<0.05)$ (Table 2).

\section{Impacts of medical insurance schemes on healthcare utilization}

Table 3 demonstrated the impacts of medical insurance schemes on healthcare utilization. Specifically, individuals covered by medical insurance schemes were more likely to receive both outpatient and inpatient services than individuals with no medical insurance schemes $(p<0.05)$. Compared with individuals covered by NRCM $\mathrm{S}$, individuals covered by URBMI were less likely to receive outpatient service $(p<0.05)$, while individuals covered by UEBMI were more likely to receive inpatient service $(p<0.05)$. There was a positive correlation between economic status and healthcare utilization, that is, the richer the individuals were, the more healthcare services were likely to be receive $(p<0.05)$. What's more, individuals older than 65 years old, living in a household of more than 2 people, with chronic diseases, fair-topoor self-reported health, or with moderate to high depression were more likely to receive both outpatient and inpatient services $(p<0.05)$. Besides, individuals with higher education status or locating in rural areas were more likely to receive outpatient service. The situation was the opposite for inpatient service utilization even the statistical results were not significant.

\section{Discussion}

We observed that outpatient service utilization increased in 2013 and then decreased in 2015, while inpatient service utilization increased over time with distinct changes. This indicated that the demands of inpatient care may still not be met. We identified that medical insurance schemes would improve healthcare utilization but different medical insurance schemes exerted various impacts on the middle-aged and elderly.

In our study, more than $60 \%$ of the individuals suffered from NCD and about $75 \%$ of the individuals did not claim good self-reported health, which indicated the grim reality that there were significant healthcare service demands [24, 25]. It was witnessed a decreasing trend in chronic disease conditions as well as poor self-reported health in contrast to the increasing healthcare service utilization. This may be partly due to improvement for chronic disease prevention and management which allowed the patients to better access the healthcare service [26]. We also found that during the study period, the average utilization rate of outpatient service and inpatient service for the middle-aged and elderly were among 19.3 and $12.7 \%$, respectively, which was consistent with Nation Health Services Survey in China conducted in 2013 (outpatient: 13.7\% 26.4\%; inpatient: $7.3 \%$ 19.9\%) [27]. Additionally, we found that the proportion of individuals covered by medical insurance 
Table 3 Determinants of healthcare utilization in the middle-aged and elderly in 2011, 2013 and 2015

\begin{tabular}{|c|c|c|c|c|c|c|c|}
\hline \multirow[t]{2}{*}{ Variables } & \multirow[t]{2}{*}{ Reference Group } & \multicolumn{3}{|c|}{ Outpatient Service Utilization } & \multicolumn{3}{|c|}{ Inpatient Service Utilization } \\
\hline & & Coefficient & SE & $p$ & Coefficient & SE & p \\
\hline Gender & Female & & & & & & \\
\hline Male & & 0.239 & 0.037 & 0.000 & -0.062 & 0.045 & 0.168 \\
\hline Age & {$[45,55]$} & & & & & & \\
\hline$(55,65]$ & & -0.008 & 0.041 & 0.837 & 0.345 & 0.052 & 0.000 \\
\hline$(65,+\infty)$ & & 0.169 & 0.049 & 0.001 & 0.739 & 0.060 & 0.000 \\
\hline Marital Status & Married or Cohabitated & & & & & & \\
\hline \multicolumn{2}{|c|}{ Divorced, Widowed, Never Married, Separated } & -0.030 & 0.056 & 0.590 & 0.011 & 0.065 & 0.869 \\
\hline Education Status & Below Primary School & & & & & & \\
\hline Primary School & & 0.067 & 0.046 & 0.143 & 0.005 & 0.055 & 0.922 \\
\hline Secondary School & & 0.094 & 0.057 & 0.101 & -0.095 & 0.070 & 0.176 \\
\hline High School and Above & & 0.148 & 0.071 & 0.036 & -0.260 & 0.087 & 0.003 \\
\hline Registration Place & Non-rural areas & & & & & & \\
\hline Rural areas & & 0.053 & 0.041 & 0.192 & -0.087 & 0.050 & 0.078 \\
\hline Region & Eastern & & & & & & \\
\hline Central & & -0.072 & 0.043 & 0.094 & 0.182 & 0.054 & 0.001 \\
\hline Western & & 0.039 & 0.043 & 0.356 & 0.398 & 0.053 & 0.000 \\
\hline Household Size & $1-2$ & & & & & & \\
\hline $3-4$ & & 0.142 & 0.040 & 0.000 & 0.126 & 0.048 & 0.008 \\
\hline$\geq 5$ & & 0.249 & 0.046 & 0.000 & 0.139 & 0.056 & 0.013 \\
\hline Chronic Diseases ${ }^{a}$ & No & & & & & & \\
\hline Yes & & 0.467 & 0.040 & 0.000 & 0.403 & 0.049 & 0.000 \\
\hline Self-Reported Health & Good & & & & & & \\
\hline Fair & & 0.630 & 0.046 & 0.000 & 0.625 & 0.059 & 0.000 \\
\hline Poor & & 1.364 & 0.055 & 0.000 & 1.584 & 0.067 & 0.000 \\
\hline Depression & Low & & & & & & \\
\hline Moderate & & 0.315 & 0.039 & 0.000 & 0.254 & 0.047 & 0.000 \\
\hline High & & 0.480 & 0.072 & 0.000 & 0.367 & 0.084 & 0.000 \\
\hline Economic Status & Quintile 1 (Poorest) & & & & & & \\
\hline Quintile 2 & & 0.243 & 0.052 & 0.000 & 0.258 & 0.068 & 0.000 \\
\hline Quintile 3 & & 0.283 & 0.053 & 0.000 & 0.609 & 0.067 & 0.000 \\
\hline Quintile 4 & & 0.317 & 0.054 & 0.000 & 0.796 & 0.067 & 0.000 \\
\hline Quintile 5 & & 0.494 & 0.056 & 0.000 & 1.058 & 0.070 & 0.000 \\
\hline Medical Insurance Schemes & NRCMS & & & & & & \\
\hline UEBMI & & 0.009 & 0.060 & 0.882 & 0.340 & 0.070 & 0.000 \\
\hline URBMI & & -0.172 & 0.072 & 0.016 & 0.136 & 0.082 & 0.095 \\
\hline PMl & & 0.024 & 0.109 & 0.827 & 0.226 & 0.131 & 0.083 \\
\hline NONE & & -0.422 & 0.077 & 0.000 & -0.545 & 0.100 & 0.000 \\
\hline
\end{tabular}

McKelvey \& Zavoina's $\mathrm{R}^{2}$ of the model used in our study were 0.1027 (outpatient service utilization) and 0.1493(inpatient service utilization)

NRCMS New Rural Cooperative Medical Insurance Scheme, UEBMI Urban Employees Basic Medical Insurance, URBMI Urban Residents' Basic Medical Insurance, PMI Private Medical Insurance

${ }^{a}$ Chronic diseases included hypertension, dyslipidemia, diabetes or high blood sugar, cancer or malignant tumor (excluding minor skin cancers), chronic lung diseases, such as chronic bronchitis, emphysema (excluding tumors, or cancer), liver disease (except fatty liver, tumors, and cancer), heart attack, stroke, kidney disease (except for tumor or cancer), stomach or other digestive disease (except for tumor or cancer), emotional, nervous, or psychiatric problems, memoryrelated disease, arthritis or rheumatism and asthma. In the surveys, people were asked whether they had ever been diagnosed with one of the listed chronic diseases and their responses were recorded 
increased in 2013 and then decreased in 2015. Above all, the majority of respondents were members of the NRCMS voluntary scheme. The decrease in medical insurance coverage in our study may be due to the lack of health literacy for the insured to reapply for their insurance schemes. Besides, individuals may choose not to renew membership because they were healthy enough or they failed to receive high-quality services as needed.

We found that individuals with a higher economic status tended to receive more outpatient service and inpatient service, which aligned with the study conducted in Gansu and Zhejiang provinces [15]. Economic status was fundamental to healthcare utilization of individuals and was one of the essential inequity elements favoring the better-off [28, 29]. Despite rapid economic growth following China's economic reforms in the late 1970s, the gap between the rich and the poor has also increased dramatically [9]. Although the expanded medical insurance could partly alleviate the burden of healthcare service, the impacts were fairly limited. The significant regional disparities in both healthcare resources and quality may impede the healthcare accessibility and affordability of individuals [30].

In our study, individuals covered by medical insurance schemes tended to receive more healthcare services. As a social security system, medical insurance schemes were established to guarantee healthcare utilization as needed and spread the risk of medical expenditures through the co-payment mechanism [31, 32]. Thus, this finding demonstrated that the expanded medical insurance schemes in China may stimulate the healthcare-seeking behaviors of the insured and unleash healthcare demands of the disadvantaged population in general, which was broadly consistent with the existing literature [33-35]. However, improved access to healthcare services may also vary among individuals with different medical insurance schemes because of the various benefit packages and the different financing schemes [36]. For instance, URBMI did not cover outpatient service so the insured had to pay for outpatient by themselves, while individuals covered by NRCMI could reimburse partial outpatient expenses. This could partly explain our finding that compared with NRCMS, URBMI decreased the likelihood of using outpatient service [11]. We also observed that individuals covered by UEBMI were more likely to receive inpatient service than their counterparts covered by NRCMS. Comparing to NRCMS, UEBMI had more generous benefit package and a higher reimbursement rate [11]. This may motivate the insured to seek healthcare services when sick with chronic or acute conditions. Lastly, the target of UEBMI was employees and retirees in urban areas, who generally had better economic conditions than people in rural areas. This might induce the insured to take advantage of healthcare services and may result in overconsumption of healthcare services [11].

However, there are several limitations subject to this study. All information about healthcare utilization, health status as well as economic status was selfreported and therefore inevitably demonstrated memory bias. Besides, the study population was not the same in three surveys (unbalanced panel data), and the benefit packages of different medical insurance schemes were expanded during the study period. This may affect the impacts of medical insurance schemes on healthcare utilization. Moreover, healthcare utilization was measured by outpatient and inpatient visits. The perspective of healthcare utilization assessment was incomprehensive to some extent. Further study should also take indicators such as the intensity of healthcare services into consideration.

\section{Conclusions}

Our findings indicated that increased medical insurance coverage can increase the likelihood of healthcare utilization, especially the inpatient service utilization. Variation of the impacts can be identified among different medical insurance schemes. Further evaluation is needed regarding whether the expanded medical insurance schemes can protect the middle-aged and elderly households from catastrophic health expenditure.

\section{Abbreviations \\ CHARLS: China Health and Retirement Longitudinal Study; NCD: Non- Communicable Diseases; NRCMS: New Rural Cooperative Medical Insurance Scheme; PMI: Private Medical Insurance; UEBMI: Urban Employees Basic Medical Insurance; URBMI: Urban Residents' Basic Medical Insurance \\ Acknowledgements \\ The authors gratefully acknowledge the national development research institute at Peking University for providing us with the CHARLS data.}

\section{Authors' contributions}

XDG and SLW conceived the study. YZ and XDG designed the study. All authors acquired and analyzed the data. YZ, HW, DV, BYN interpreted the findings. YZ and HW wrote the first draft of the manuscript. SLW drafted subsequent versions. All authors critically reviewed this article and approved the final version.

\section{Funding}

The authors disclosed receipt of the following financial support for the research, authorship, and publication of this article: This work was supported by the National Natural Science Foundation of China [Grant No. 71774005].

The funders had no role in study design, data collection and analysis, decision to publish, or preparation of the manuscript.

\section{Availability of data and materials}

The datasets supporting the conclusions of this article are available in China Health and Retirement Longitudinal Study (http://charls.pku.edu.cn/). Researchers can log on the website to register by signing the agreement and provide real personal information for reviewing. After completing the registration, researchers could apply for publicly available datasets respectively, and the application will be reviewed and approved in 3 working days. If researchers have any questions or suggestions, they can contact CHARLS by email charls_info@pku.edu.cn or by phone 86-400-6101866 or 86-(0)10-62767425. 


\section{Ethics approval and consent to participate}

Ethical approval for this study was not necessary because it was based exclusively on publicly available data, CHARLS, and the study subjects were not directly approached.

\section{Consent for publication}

Not applicable.

\section{Competing interests}

The authors declare that they have no competing interests.

\section{Author details}

'Department of Pharmacy Administration and Clinical Pharmacy, School of Pharmaceutical Sciences, Peking University, No.38 Xueyuan Road, Haidian District, Beijing 100191, China. ${ }^{2}$ Center for Strategic Studies, Chinese Academy of Engineering, No.2 Bingjiaokou HuTong, Xicheng District, Beijing 100088, China. ${ }^{3}$ School of Medicine, Tsinghua University, No.30 Shuangqing Road, Haidian District, Beijing 100084, China. ${ }^{4}$ School of Health Humanities, Peking University, No.38 Xueyuan Road, Haidian District, Beijing 100191, China. ${ }^{5}$ International Research Center for Medicinal Administration, Peking University, No.38 Xueyuan Road, Haidian District, Beijing 100191, China. ${ }^{6}$ Department of Population Medicine, Harvard Medical School and Harvard Pilgrim Health Care Institute, 133 Brookline Avenue, Boston, MA 02215, USA.

\section{Received: 22 July 2019 Accepted: 8 July 2020}

Published online: 14 July 2020

\section{References}

1. Chatterji S, Kowal P, Mathers C, Naidoo N, Verdes E, Smith JP, et al. The health of aging populations in China and India. Health Aff (Millwood). 2008; 27(4):1052-63.

2. China Statistical Yearbook 2018 [Internet]. http://www.stats.gov.cn/tjsj/ndsj/2 018/indexeh.htm. Accessed 25 Feb 2019.

3. Lozano R, Naghavi M, Foreman K, Lim S, Shibuya K, Aboyans V, et al. Global and regional mortality from 235 causes of death for 20 age groups in 1990 and 2010: a systematic analysis for the global burden of disease study 2010. Lancet. 2012;380:2095-128.

4. World Report on Ageing and Health [Internet]. Geneva: World Health Organization; 2015. https://www.who.int/ageing/events/world-report-2015launch/en/. Accessed 25 Feb 2019.

5. National Health and Family Planning Commission of People's Republic of China. China health statistics yearbook. Beijing: Peking Union Medical College Press; 2017.

6. Chen W, Gao R, Liu L, Zhu M, Wang W, Wang Y, et al. Summary of China cardiovascular disease report 2017. Chin Circ J. 2018;33(1):1-8 (in Chinese).

7. Mendis S, Armstrong T, Bettcher D, Branca F, Lauer J. Cecile Mace, et al. global status report on noncommunicable diseases. Geneva: World Health Organization; 2014.

8. Eichengreen B, Park D, Shin K. When fast growing economies slow down: international evidence and implications for the People's republic of China. Manila: Asian Development Bank; 2011. http://www.ssrn.com/abstract=1883962. Accessed 25 Feb 2019.

9. Benjamin D, Brandt L, Giles J, Wang S. Income inequality during China's economic transition. In: Brandt L, Rawski TG, editors. China's great economic transformation. Cambridge: Cambridge University Press; 2008. p. 729-75.

10. Hu R, Shen C, Zou H-f. Health care system reform in China: issues, challenges and options. Beijing: China Economics and Management Academy, Central University of Finance and Economics; 2011.

11. Qingyue M, Yang H, Wen C, Qiang S, Xiaoyun L. People's republic of China health system review. Manila: World Health Organization. Regional Office for the Western Pacific; 2015. http://www.who.int/iris/handle/10665/208229. Accessed 25 Feb 2019.

12. National Health and Family Planning Commission of People's Republic of China. China health statistics yearbook. Beijing: Peking Union Medical College Press; 2009.

13. National Health and Family Planning Commission of People's Republic of China. China health statistics yearbook. Beijing: Peking Union Medical College Press; 2012.

14. National Health and Family Planning Commission of People's Republic of China. China health statistics yearbook. Beijing: Peking Union Medical College Press; 2016.
15. Wang Y, Wang J, Maitland E, Zhao Y, Nicholas S, Lu M. Growing old before growing rich: inequality in health service utilization among the mid-aged and elderly in Gansu and Zhejiang provinces. China BMC Health Serv Res. 2012:12:302.

16. Liu H, Zhao Z. Impact of China's urban resident basic medical insurance on health care utilization and expenditure. Bonn: The Institute for the Study of Labor Discussion Paper; 2012.

17. Gong $\mathrm{CH}$, Kendig $\mathrm{H}, \mathrm{He} \mathrm{X}$. Factors predicting health services use among older people in China: an analysis of the China health and retirement longitudinal study 2013. BMC Health Serv Res. 2016;16:63.

18. Ren J, Ding D, Wu Q, Liu C, Hao Y, Cui Y, et al. Financial Affordability, Health Insurance, and Use of Health Care Services by the Elderly: Findings From the China Health and Retirement Longitudinal Study. Asia Pac J Public Health. 2019;31:510-21.

19. CHARLS . http://charls.pku.edu.cn/en. Accessed 20 Jan 2018.

20. Wang Z, Li X, Chen M. Catastrophic health expenditures and its inequality in elderly households with chronic disease patients in China. Int J Equity Health. 2015;14(1):8

21. Jin $Y$, Hou Z, Zhang D. Determinants of Health Insurance Coverage among People Aged 45 and over in China: Who Buys Public, Private and Multiple Insurance. PLoS One. 2016;11(8):e0161774.

22. National Data [Internet]. http://data.stats.gov.cn/english/. Accessed 20 Jan 2018

23. The Central Bank of the People's Republic of China [Internet]. https://data worldbank.org/. Accessed 20 Jan 2018.

24. Geitona M, Zavras D, Kyriopoulos J. Determinants of healthcare utilization in Greece: implications for decision-making. Eur J Gen Pract. 2007;13(3):144-50.

25. Dey DK, Mishra V. Determinants of choice of healthcare services utilization: empirical evidence from India. Indian J Community Health. 2014;26(4):356-63.

26. Medium-and long-term planning for prevention and treatment of noncommunicable disease in China [Internet]. http://www.gov.cn/zhengce/ content/2017-02/14/content_5167886.htm. Accessed 12 Feb 2020.

27. National Health Commission of the People's Republic of China. Nation Health Services Survey in China. Beijing; 2013.

28. Fu X, Sun N, Xu F, Li J, Tang Q, He J, et al. Influencing Factors of Inequity in Health Services Utilization Among the Elderly in China. Int J Equity Health. 2018;17:144.

29. Penning MJ, Zheng C. Income inequities in health care utilization among adults aged 50 and older. Can J Aging. 2016;35(1):55-69.

30. Meng Q. Developing and implementing equity-promoting health care policies in China. Beijing: Health Systems Knowledge Network of the World Health Organization's Commission on Social Determinants of Health; 2007.

31. Price DW, Swaney RE III, Xu S, Goodspeed JR, Steiner JF. Care of Common Medical Conditions in a managed care program for uninsured adults. Permanente J. 2003;7(1):27-35.

32. Donelan K, Blendon RJ, Hill CA, Hoffman C, Rowland D. Whatever happened to the health insurance crisis in the United States? JAMA. 1996;276(16): 1346-50.

33. Wagstaff $A$, Lindelow $M$, Jun $G$, Ling $X$, Juncheng $Q$. Extending health insurance to the rural population: an impact evaluation of China's new cooperative medical scheme. J Health Econ. 2009;28(1):1-19.

34. Wagstaff $A$, Pradhan M. Health insurance impacts on health and nonmedical consumption in a developing country. Washington DC: The World Bank; 2005.

35. Aron-Dine A, Einav L, Finkelstein A, Cullen M. Moral Hazard in health insurance: do dynamic incentives matter? Rev Econ Stat. 2015;97(4):725-41.

36. Fang $\mathrm{H}$, Meng Q, Rizzo JA. Do different health insurance plans in China create disparities in health care utilization and expenditures. Int J Appl Econ. 2014;11(1):1-18.

\section{Publisher's Note}

Springer Nature remains neutral with regard to jurisdictional claims in published maps and institutional affiliations. 